\title{
TOWARDS DIGITAL TWIN DRIVEN CULTURAL HERITAGE MANAGEMENT: A HBIM-BASED WORKFLOW FOR ENERGY IMPROVEMENT OF MODERN BUILDINGS
}

\author{
A. Massafra ${ }^{1}$, G. Predari ${ }^{1}$, R. Gulli ${ }^{1}$ \\ ${ }^{1}$ Department of Architecture, University of Bologna, Bologna, Italy - (angelo.massafra2, giorgia.predari, riccardo.gulli)@unibo.it
}

Commission V, WG V/7

\begin{abstract}
KEY WORDS: Cultural heritage, Energy improvement, Digital Twins (DT), Heritage Building Information Modeling (HBIM), Building Energy Modeling (BEM), Building Performance Simulation (BPS), International Foundation Classes (IFC), Multi-criteria analysis.
\end{abstract}

\begin{abstract}
:
Europe has numerous historic buildings that need to become more energy-efficient, which need permanent maintenance and refurbishment to fulfill sustainability and use requirements. Asset owners and asset managers need to adopt new strategies to protect listed buildings while optimizing costs and benefits during their life cycle. In this sense, the digital transition proves to be a moment to seize for opening new scenarios. The Digital Twin paradigm promises to be valuable for enabling the sustainable knowledge, conservation, restoration, and management of built assets and solving the dilemma about protecting the architectural identity of these buildings while adapting them to the functional and performance requirements dictated by the regulatory framework. This study proposes a workflow that integrates Heritage Building Information Modeling (HBIM) and Building Performance Simulation (BPS) tools for data-driving the energy improvement of Italian listed modern buildings built between the 1920s and 1960s. After acquiring information about the building, the HBIM model and the Building Energy Model (BEM) are realized based on the International Foundation Classes (IFC) standard. Energy intervention measures are defined, construction costs are computed, and benefits during the intervention life cycle are predicted in thermal demand. Finally, an expeditious multi-criteria analysis allows for comparing different intervention combinations and indicating the optimal solution for the energy improvement of the building concerning energy, economic, and financial issues. These outcomes represent the first step towards realizing a dynamic, accessible, and sharable Digital Twin.
\end{abstract}

\section{INTRODUCTION}

Climate change is one of the main threats to the natural and anthropic environment. According to the 2030 Agenda for Sustainable Development (UN General Assembly, 2015), although cities and metropolitan areas occupy only $3 \%$ of Earth's surface, they contribute about $60 \%$ of global Gross Domestic Product (GDP). They also account for about $70 \%$ of global carbon emissions and over $60 \%$ of energy use. These data are alarming when combined with the expected world population growth, which will progressively require new energy and soil consumption.

To face these issues, international organizations have been undertaking several initiatives. European member states are implementing guidelines on the use of renewable energy sources and the energy performance of the buildings. Meanwhile, the European Commission has financed many projects to promote zero or even positive-energy buildings. This policy has led to the construction of new buildings with very high performance.

Nevertheless, there is a strong need to improve the energy performance of existing buildings to decrease their impact on $\mathrm{CO}_{2}$ emission and climate change. Europe has many historic buildings that require becoming more energy-efficient while needing also permanent maintenance and refurbishment to fulfill sustainability and use requirements.

In Italy, about $60 \%$ of existing buildings date back to the period between the 1920s and 1960s, the so-called "Modern age". This point is critical since modern architectures are generally incompatible with environmental and economic performance requirements dictated by current regulations and with sustainable use and management conditions. Although they are affected by some intrinsic weaknesses related to their construction type, most of them should be protected because of their historical, architectural, and symbolic value (Poretti, 2008).

In order to achieve the goal of the sustainable management of the built heritage, asset owners and asset managers need to adopt new strategies to protect modern buildings while optimizing costs and benefits during their life cycle. The digital transition proves to be a moment to seize for opening new scenarios. It can provide suitable responses to current critical problems, reduce the soil consumption rate, and improve the performance and conservation state of existing buildings.

The Digital Twin (DT) paradigm (Grieves, 2019), imported by the Smart Manufacturing domain (Tao et al., 2019), promises to be valuable for enabling the sustainable knowledge, conservation, restoration, and management of built assets. It could be an innovative management model, with essential importance concerning the dilemma about protecting the architectural identity of modern buildings while adapting them to the functional requirements dictated by the regulatory framework, the needs of environmental protection, and the users' comfort. In addition, basing DT on approaches such as Building Information Modeling (BIM), or Heritage Building Information Modeling (HBIM) (Murphy et al., 2009), can help save costs and reduce energy consumption in historic buildings, mainly if open formats are used for information exchange and transformation during the building lifecycle. On the one hand, open-BIM 
methods can help to acquire, structure, and archive reliable data, which often these buildings lack, so that they can be used independently from the software that has generated it in the future (buildingSMART Italy, 2021). On the other hand, integrating BIM with Building Performance Simulation (BPS) tools can permit the processing of advanced information on the state of the built assets, such as buildings energy behavior, and support owners' decision-making in intervention planning and renovation design since the preliminary phases.

For these reasons, a research project is being carried out to develop data-driven procedures for supporting the renovation design of some modern listed buildings of the University of Bologna and enabling their performance-based management. This paper focuses on the first step of this study. An HBIM-based workflow is presented for evaluating energy improvement strategies on the Institute of Mathematics of Bologna. The workflow addresses the subject of the interoperability between BIM and BPS data, models, and software by using, albeit with some limits, the International Foundation Classes (IFC) standard for performing the energy assessment of the building and the intervention solutions. In the workflow, BIM and Building Energy Model (BEM) data are used to calculate the annual energy demand for the building heating and compute investment and lifecycle costs related to different intervention strategies. This information is then considered in a simplified multi-criteria analysis to identify the optimal renovation solutions for the energy improvement of the building regarding economic, energy, and environmental issues.

\section{BACKGROUND}

\subsection{Modern Construction in Italy}

The study of Modern Architecture has generally been driven in the literature by a twofold reason. The first is to rescue from oblivion an entire generation of architects and works obscured by historical prejudices, placing them in a correct perspective in the History of Architecture and Engineering (Iori, 2014; Zevi, 2004). The second refers to the research on the material culture of the 20th-century construction to reach an in-depth knowledge of the technical issues of modern buildings and enable their aware protection, conservation, and restoration.

A high formal innovation and marked technical experimentation characterized the Italian architectural heritage of the 20th century (Poretti, 2008). In the first phase of Modern Italian Construction, the School of Applications achieved remarkable progress in the scientific and theoretical fields by studying the inapplicability of elastic theory to the anisotropic material, deepening research on plastic behavior, and understanding cracking and breaking phenomena (Mochi and Predari, 2012). Meanwhile, the construction system of reinforced concrete was widely and rapidly spreading, with the particularity that Italian reinforced concrete frames initially consisted of masonry-mixed structures. Then, a following period of great technical experimentation and test of reinforced concrete technique occurred in construction sites of public works from the 1920s to the 1940s.

Regarding the building envelopes, the new construction system did not instantly caused the refusal of the traditional language linked to wall construction from the figurative point of view. However, the insertion of structural frames in buildings began to change the style of masonry systems, leading to their decisive revision. During the autarchy, the major works confronted with the need of representing the autarchic ideology through stone facings that hide structural systems. On the contrary, after World War II, the mixed masonry construction started to manifest its figurative and spatial autonomy, defining a trend that historiography usually categorizes as Architectural Realism (Poretti, 2008).

The multidisciplinary knowledge of modern buildings plays a crucial role in conserving Italian heritage since this building type is widely spread throughout the peninsula. However, conservation processes are not trivial. Nowadays, modern heritage shows a remarkable symbolic, historical, and architectural value. Still, it is affected by features genetically intrinsic to its construction type, and many of them represent the results of the experimental practices of 20th-century building sites. All these architectures were built before the 1970s without a proper regulatory framework on energy behavior and seismic structural safety.

At present, modern buildings are recently under the protection rules dictated by the Italian Code of Cultural Heritage and Landscape. At the same time, they present evident incompatibility with the environmental, economic, and functional needs currently required for their sustainable use and management, which are directly related to major owners as public administrations.

On the one hand, the issue concerns buildings and their state of maintenance and performance. On the other hand, owners need the functional compatibility of built assets to respect a demanding framework changing over time, both in logistics and physical refurbishment. Therefore, it is essential to enable protection processes to preserve the cultural and social values of these buildings while allowing their practical use, optimizing costs and benefits during their lifecycle, and complying with environmental issues.

\subsection{Digital transition for the built environment}

The growing importance of data and the exponential development of new technologies has triggered the digital transition in recent years. At present, it is time to seize opportunities in various domains; among them, the construction sector and the built environment management are crucial for reaching the goal of the sustainable development of cities and communities.

The literature agrees that the potentials, methods, and instances of the coming digital transition can enable the development of protocols to optimize the processes of knowledge, conservation, restoration, and management of the built heritage (Osello et al., 2018). However, there are significant obstacles in digitizing the Architecture, Engineering and Construction Industry.

Firstly, traditional administrative practices diverge from those required by adopting digital processes for planning interventions, for the use, the management, and the maintenance of built assets (Di Giuda and Pattini, 2021). Then there are additional difficulties in Cultural Heritage preservation (Korro Bañuelos et al., 2021). Historical architectures are often unique objects whose information is challenging to find since it is usually fragmented in numerous paper archives.

Secondly, it is challenging to predict immediate and tangible benefits for owners since authorities, contractors, and professionals often lack synergetic and transparent strategic thinking. The advantages of digital approaches over usual ones in cost, time, quality, and control must also be transformed into organizational benefits for all the involved parties. 
Thirdly, the application of digital technology in these areas offers many opportunities, but they are not yet defined into a scientific methodology (Succar and Poirier, 2020). It is thus necessary to formulate new theoretical and conceptual frameworks in parallel with the development of new digital practices to drive the sustainable informed management of built heritage by administrations.

In response to the frenetic pace imposed by digitization, the international scientific community has been introducing new digital paradigms in the construction industry, such as Building Information Modeling (BIM), Heritage Building Information Modeling (HBIM), Smart and Cognitive Buildings, Digital Twins (DT), Internet of Things (IoT), and Artificial Intelligence (AI). Various national and international institutions have proposed standards, protocols, specifications, and regulations. The software industry has also contributed to the development of new digital practices. BIM authoring tools have been introduced and updated, and their interoperability with Building Performance Simulation (BPS) software has been implemented to develop shared digital environments. The idea of open tools, data, and models is now widely accepted.

Initiatives and research have been undertaken at the national or international level. All these contributions underline that it is strictly necessary to develop top-level conceptual constructs to promote the digital innovation of the sector. In addition, smart contracts and blockchain are being introduced to make all information exchanges between the parties involved transparent and reliable (Lee et al., 2021).

In order to address a sustainable digital transition process, two different levels of knowledge must be investigated. The former must define new ontological data models to enable the organic development of new digital practices, activate standardized and shared information modeling protocols, and encourage the involvement of all operators in the computerized technical management of built assets. The latter must provide examples of good digital practices for the built heritage management by applying specific workflows to significant case studies.

\section{CASE STUDY}

\subsection{The Alma Mater's building stock}

Public administrations are often affected by a series of organizational constraints that do not allow fulfilling the changing requirements over the lifecycle of the buildings. This incapacity often translates into a waste of public financial resources.

The emergency condition produced by the pandemic has made this issue evident, and it has strongly promoted the transformation of the current organizational structures of asset managers. On the one hand, the recent changes in the demanding framework require the rethinking of spaces for work in presence in terms of function, comfort, and consumption. On the other hand, more sustainable management of built heritage is needed to improve the performances of existing public buildings in terms of energy and environmental impact.

Given this context, it was decided to focus on the vast building stock of the Alma Mater Studiorum University of Bologna. It is a significant case study for twofold reasons. Firstly, many modern buildings of the University have high architectural and historical values that need to be maintained. Secondly, the University of Bologna ranks first as an owner of public real estate assets in the Emilia-Romagna region with about 1 million square meters of area and about 70,000 inhabitants. Therefore, this building set can be considered meaningful both at the building and the city scale.

\subsection{The Institute of Mathematics of Bologna}

Within this background, a paradigmatic case study was identified. The Institute of Mathematics of Bologna, designed by Giovanni Michelucci, is a valuable example of modern architecture built between 1955 and 1965 (Inglese and Ferrari, 2010).

The building is located in the University area of Bologna near the ancient walls that enclosed the medieval city. It consists of two blocks. Block A is 24 meters high and it spreads over ten staggered levels, with an average floor area of 490 square meters. It hosts offices for professors and researchers, small classrooms, a library, and a book warehouse. Block B is a 36-meters-high tower with eight floors with an average floor area of 365 square meters. It hosts large step-shaped classrooms and some offices. The total area covered by the building is 900 square meters.

The most characterizing architectural element of the main façade is the portico that evokes the medieval architecture of Bologna with fork-shaped pillars.

After the construction of the building, some maintenance was performed. In the 1980s, an external staircase was added for fire safety, then the asbestos roof of Block B was replaced with fibercement sheets due to its degradation.

This construction currently presents some critical structural and energy performance issues since the Insititute was built before the first Italian regulation on structural seismic design and energy efficiency of buildings.

The building structure is made of reinforced concrete pillars and beams, with hollow brick and roof slabs of reinforced concrete with air chamber. It presents some critical features that are common to Italian modern buildings, such as the organization on monodirectional frames, smooth reinforcing steel bars, low shear resistance in correspondence of beam-pillar nodes, high deformability of floor slabs, and variability of concrete compressive strength.

The building envelope is generally characterized by a high Uvalue and, therefore, poor energy performance for heating. The facades consist of two layers of solid bricks spaced by an air chamber with variable thickness, finished by mortar and $2-\mathrm{cm}-$ thick brick slats. The windows, almost all dating back to the original construction of the building, have anodized aluminum frames and 3 -cm-thick single-glass. The roofing envelopes consist of reinforced concrete slabs covered by polystyrene, bitumen, and tiles in Block A and fiber-cement sheets in Block B.

\section{METHODS}

\subsection{The HBIM-based workflow}

In recent years, BIM is gaining importance in the Cultural Heritage domain. The concept of developing virtual models for historical buildings that go beyond a simple geometrical representation is becoming increasingly common. HBIM could be a new methodology for managing built assets and sharing its information with building lifecycle operators. Once building data is structured and integrated into digital models, the latter could be used in platforms where stakeholders can manage the data collaboratively. In addition, integrating BIM with performance 
simulations could create a deeper knowledge of buildings behavior and allow for data-driven renovation strategies.

In this study, an HBIM-based workflow was systematized for supporting the preliminary design of energy retrofit interventions on the presented case study. In particular, the envelope system was investigated as its improvement is supposed essential to reduce plant energy consumption and $\mathrm{CO}_{2}$ emissions during the operation and maintenance $(\mathrm{O} \& \mathrm{M})$.

The procedure develops following a four-step method of investigation (Figure 1). First, in the documental phase, information about the building is acquired through historicalarchival studies, digital surveys, and in-situ inspections or is shared with the building administrators. Then the computational phase involves the realization of the HBIM Model and the Building Energy Model (BEM), both based on IFC format. After that, in the predictive phase, energy intervention measures are defined, construction costs are computed through BIM data, and benefits during the intervention lifecycle are predicted in terms of thermal demand and energy costs for heating through BEM data. An expeditious multi-criteria analysis compares different intervention combinations and indicates the optimal solution for the energy improvement of the building measures concerning economic, energy, and environmental issues. In future research developments, the operational phase will monitor dynamic parameters related to the chosen intervention actions by implementing an IoT sensor network and, therefore, of a dynamic Digital Twin.

\section{DOCUMENTAL PHASE}

Archival/bibliographic research

Digital survey

In-situ investigations

Information sharing with administrators

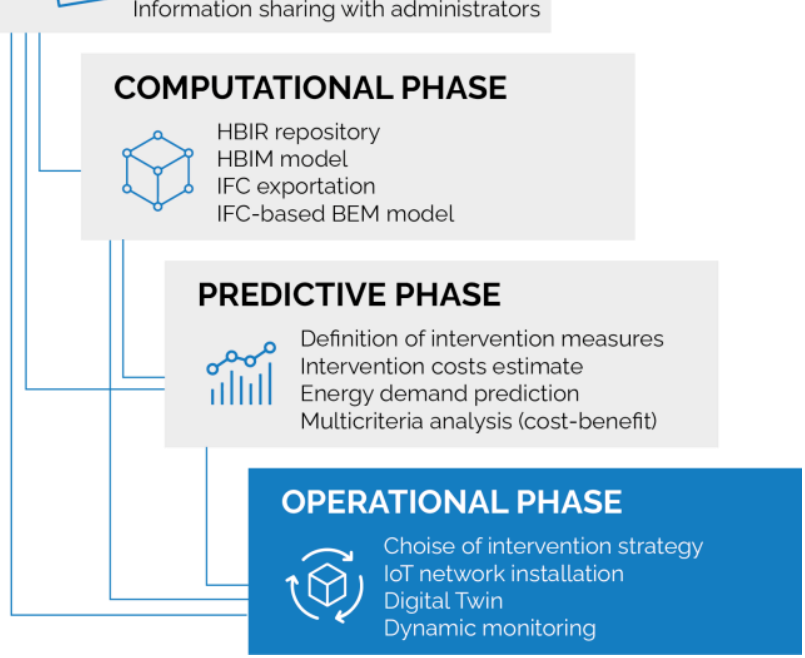

Figure 1. The HBIM-based workflow for the energy improvement of modern buildings.

\subsection{Definition of information requirements}

Although issues related to the accuracy of digital 3D reconstructions of non-regular geometries for historic buildings have attracted most researchers, the main aim of HBIM processes is integrating the knowledge of the heritage in data models. Therefore, BIM models must reflect the existing building as much as possible in terms of geometrical and information completeness (Jouan and Hallot, 2019).
Geometry accuracy is only one aspect that interests a BIM model or a DT. When adopting a BIM-based Digital Twin, precise criteria need to be also defined inherently to the uses of BIM (Kreider and Messner, 2013), the level of knowledge of the data integrated into the models and elaborated in the analyses (Castellano-Román and Pinto-Puerto, 2019), and the capability level of the predictive models to be used to support building management decisions. It is better to define all these information requirements from the preliminary phase of the digitization process to take all the advantages led by the BIM approach in the following phases.

According to (buildingSMART Italy, 2021), uses are nonoptional but prescriptive to achieve the desired results from the beginning, above all for economic issues. If decisions are taken with the correct information, future problems will be solved easier in terms of time and cost. Nevertheless, the definition of BIM uses is not a straightforward operation since many different BIM uses could be involved in realizing a building digital twin, and they could be incompatible with each other. In addition, all the uses need to be agreed upon with all the stakeholders.

The maturity of the analytical models and the information fidelity are two other important metrics to be defined before making digital models. Maturity models have been revealed in the software industry to improve software development and maintenance capabilities. For DT and BIM, maturity levels define a set of progressive steps that emphasize the capabilities required to achieve a mature technology solution. According to (Gerber et al., 2019, p. 21), five maturity levels can be defined based on the degree of autonomy, intelligence, learning, and fidelity of the digital systems: descriptive, informative, predictive, comprehensive, and transformative. The concept of fidelity refers to information in its triple nature of data, document, or model and concerns the reliability of data or sources and actors that provide it. The more faithful a piece of information is, the better it describes a specific characteristic of the built asset. In the case of existing buildings, the fidelity level of data is a critical metric to be defined since one of the main critical issues encountered in the digitization processes of a protected historical asset is the accessibility of the data. Data acquisition processes are usually resource-intensive in terms of effort and cost and are difficult because of the fragmentation of information in different physical and digital archives. There are no univocal cataloging systems for documents, and the parties involved do not always collaborate through established practices.

For these reasons, data acquisition must be driven by specific information uses, and information reliability must be certified when information is exchanged by associating Levels of Knowledge (LOK) and Levels of Information Need (LoIN).

\subsection{Documental phase}

Extensive documentation about the asset's current condition is generally required at the beginning of a renovation project. Therefore, it is crucial to define the procedure for acquiring all data regarding the selected information uses at an early stage.

When dealing with historic heritage, archival research is fundamental to know the construction features of the building, and it can help identify properties and characteristics that otherwise would be difficult to find except by destructive tests. The survey of the building is then necessary to produce geometrically-reliable digital models since the drawings archived by authority offices could often differ from the actual status of the building. During the pre-design phase, it is also crucial to 
gather any helpful information to better evaluate the energy improvement strategies as well as all the documents related to regulating authority permissions, previous intervention, any change in use, the actual use of the buildings, the actual consumption of the energy, the thermal properties of both opaque and transparent surfaces (buildingSMART Italy, 2021).

In order to acquire valuable information about the current state of the Institute of Mathematics, several preliminary in-situ investigations were carried out. At the same time, historical and archival research was performed in public and private archives to identify the construction characteristics of the building. Consulting the construction site documents and photographs allowed for defining, for instance, the stratigraphies of the opaque and transparent envelope. Subsequently, asset managers were consulted to provide data about the geometric characteristics of the building, and the acquired plans and sections were verified on-site and modified where necessary with the help of a photogrammetric survey of the external facades. After having defined the envelope stratigraphy, the thermal attributes of the materials have been determined according to the reference standards or studies carried out on buildings with similar construction characteristics and age (Intelligent Energy Europe -IEE-, 2012). Other important information was collected by administrators regarding the actual use of the different zones of the building, the temporal duration of the uses, the energy bills of the last years, and energy costs.

\subsection{Computational phase}

4.4.1 HBIM repository and HBIM libraries: A Heritage Building Information Repository (HBIR) of the Institute of Mathematics was created to host the collected documentary records. It was helpful to quickly provide reliable information about the current condition of the building while modeling.

HBIM libraries: Then, a repertoire of digital BIM objects grouped in libraries with common shared parameters was developed to be used for the HBIM model and validated for the energy analyses of the building envelope.

This repertoire also represents the basis for developing a Data Dictionary of the Modern Construction in Bologna: a database of properties linked to digital objects that can be shared between different domains and used as standardized information sets to collect building data whenever dealing with such facilities.

4.4.2 HBIM model: The HBIM model of the Institute of Mathematics was so created in Autodesk Revit (Figure 2), employing the previously developed BIM libraries and evaluating the digital metrics described above. It was decided to finalize the BIM uses for energy modeling, cost scheduling, and geometrical modeling of the building digital twin that will be developed in the next research steps.

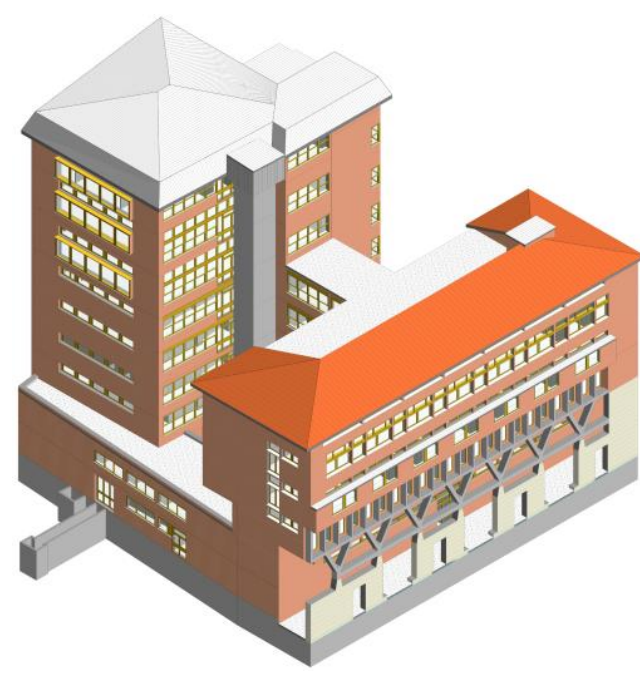

Figure 2. The HBIM model of the Institute of Mathematics.

In order to allow new BIM uses in the future and to report all the relevant geometric data of the building, the HBIM model was composed of two different models. The first one, used in the proposed energy assessment workflow, integrates the geometric data of the building envelope with the information related to the functions performed in the building, the construction characteristics of its envelope, and the thermal properties of the materials. Its semantic structure contains ifcWalls, ifcWindows, ifcRoof, ifcSlab, and ifcSpace elements. Particular attention was paid to the development of windows and envelope components in the perspective of their use in a BEM environment. The second model pertains to the geometries of the structural components. It will be enriched with helpful information for the structural assessment of the building, such as the strength of the materials and the quantity of reinforcing bars. It consists of ifcColumn, ifcBeam, ifcStair and ifcSlab elements.

For the purpose of this work, the BIM model was realized with a view to integrate it with BEM analysis for producing a predictivelevel digital system, which means a digital system able to provide predictive maintenance, analytics, and insights (Gerber et al., 2019). Also the level of the geometric accuracy of the BIM model was chosen accordingly to the aims of the workflow. A LOK300 level of knowledge was chosen among that proposed by (Castellano-Román and Pinto-Puerto, 2019) to represent the HBIM elements and their information. This level of knowledge allows for advanced research on building envelope to perform the preliminary design of its energy improvement.

4.4.3 IFC export: Various research papers investigated the interoperability between BIM and BEM and found that BIMbased building energy modeling development is still at the initial stage (Bonomolo et al., 2021). Although the aim should be to achieve the energy data of the building with a unique model, it is often necessary to create a second digital model, the Building Energy Model (BEM).

The choice of the exchange formats tools is fundamental as it must support objects to describe the complete building systems, individual components, the hierarchy of the components, their functional and topological relationships, interdependencies, and energy-related parameters or characteristics.

Generally, the data transmission between BIM and BEM is possible through two types of file formats. The first is IFC (Industry Foundation Classes), and the second is gbXML (Green 
Building Extensible Markup Language). Both have significant advantages. On the one hand, IFC is the standard format for information exchange in BIM modeling as it is the only certified format. In IFC, most of the information concerning a building can be structured except for the energy analysis data. While the gbXML is a format based on IFC that can also contain all the energetic information. On the other hand, the IFC schema is more extensive and complex than gbXML. In addition to a good geometric representation, it supports semantic data enrichment and could provide a single basis for exchanging various types of information during the building lifecycle (Porsani et al., 2021).

In this research, IFC-based standards have been used to describe information exchange from the BIM authoring software to the BEM modeling software. The IFC file of the Institute of Mathematics was exported from Autodesk Revit via IFC4 design transfer view and imported into Cypetherm C.E. for energy analysis, following the workflow proposed by (Porsani et al., 2021).

4.4.3 BEM model: Before importing the IFC file into the BEM software, the IFC Builder software was used to generate an analytical model and check the extracted data, such as dimensions, geometry, and IFC object types.

Despite good geometric compatibility in the exchange of information, the tool proved to be unsuitable in the bidirectional exchange of energy information since it was necessary to manually copy some data from BIM to BEM, such as thermal properties of materials and construction systems. Any lack of information was integrated for a preliminary feasibility study, such as energy requirements for each zone, legislation requirements and the specific geographical climate data. It was also necessary to simplify the geometry of the roof and some rooms. Many problems were related to the staggered levels of the building that do not fit into the logic of BEM software.

The energy analysis of the building was performed in Cypetherm C.E. according to the Italian regulation (UNI/TS 11300). With the collected information, an ante-operam simulation was carried out in order to evaluate the energy behavior of the building, defining its annual energy demand for heating and its energy class. Results were then calibrated on the actual energy bills encountered by the building in the last three years.

\subsection{Predictive phase}

Preliminary design: improving the energy performance of existing buildings could be very challenging, mainly if they are listed. In this case, some usual energy improvement solutions may not be appropriate to preserve the architectural value of the building, such as the external insulation of façades. Moreover, preliminary considerations about life cycle costs, energy consumption, intervention cost estimation, and environmental impact should be quantified to meet owners' needs and sustainability requirements.

This point is crucial for achieving the performance-based management of built heritage. Asset managers would need to numerically evaluate the comparison between the built status and the improved designed status by addressing procedures driven by verifiable and monitorable data even in the post-intervention phase. This procedure could be carried out faster and better if supported by high-quality BEM models.

Five intervention measures to improve the energy class of the Institute of Mathematics were identified concerning the building envelope. They are the insulation of the roof (A), the insulation of the infill walls by blowing cellulose fiber (B1) or polyurethane foam (B2), the replacement of the windows (C1), or their improvement $(\mathrm{C} 2)$.

Then, all the possible combinations of the intervention measures were investigated and, for each combination, a post-operam energy simulation was run in Cypetherm C.E. to calculate the degree of energy improvement in terms of heat demand. Intervention costs were also computed by using the BIM model in Autodesk Revit, supported by DiRoots plug-ins for scheduling.

4.5.1 Multicriteria analysis: the primary outcome of the predictive phase consists of a multi-criteria analysis that helps identify the optimal intervention combinations from the environmental, economic, and financial points of view and define various intervention strategies by comparing five parameters related to the intervention solutions, which are annual heat energy demand (ED), investment costs (IC), lifecycle costs (LCC), payback period (PBP), and upgrade level (UL) (Figure 3).

Energy demand (ED) refers to the annual energy consumption for heating predicted for the renovated building. ED was calculated by using BEM models calibrated on the energy bills in the last three years.

Intervention costs (IC) were computed through the BIM data, including materials, construction site, and labor costs.

Lifecycle costs (LCC) related to the interventions were defined through the simplified approach proposed by (Hong et al., 2021) as the sum of investment costs (IC) and net present values (NPV), assuming, for simplification, the interest rate equal to the rate of energy price increase per year.

$$
L C C=I C+N P V
$$

where $\quad L C C=$ lifecycle costs

$$
I C=\text { intervention costs }
$$

$N P V=$ net present values

$N P V=E C d \times N$,

where $\quad E C d=$ predicted annual energy consumption costs $N=$ building service life

The building service life $(\mathrm{N})$ was assumed equal to 30 years as suggested by (ENEA, 2017).

The payback period (PBP) refers to the amount of time needed to recover the cost of an investment or the length of time an investor needs to reach a break-even point. It was calculated as:

$$
P B P=I C /(E C a-E C d),
$$

where $\quad E C a=$ actual annual energy consumption costs of the existing building (by bills)

Finally, the upgrade level (UL) was defined by matching IC values, sorting them in ascending order, and varying them from 0 to 100 . For instance, a null UL value is assigned to the strategy with no incurred IC (i.e. no retrofitting), while a $100 \mathrm{UL}$ value is assigned to the strategy with the highest IC. 


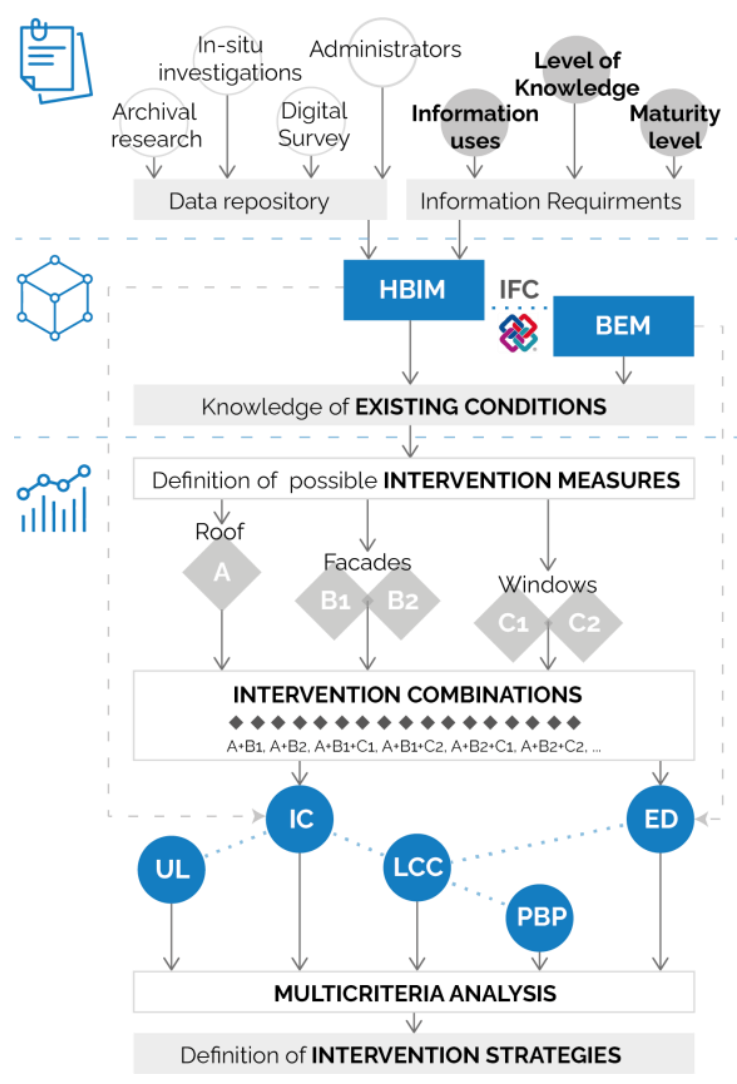

Figure 3. The data flow for determining the five parameters investigated in the multicriteria analysis.

\section{RESULTS}

The results of the multi-criteria analysis are represented in Figure 4. All values are reported from 0 to 100 in comparative terms of benefits, where 0 is the interventions combination that expresses fewer benefits and 100 is the one that expresses most benefits with reference to a specific parameter. For example, intervention combination with fewer energy benefits corresponds to null ED, while 100 is assigned for the most energy-efficient combination.

With reference to the analyzed case study, the investigation results show that energy retrofit interventions are of outmost importance not only for reducing the energy demand, and so the environmental impact, but also for reducing the operating costs during the building life cycle. By looking at the graph in Figure 4 , it is possible to observe that high intervention costs (IC) can significantly reduce energy costs and so lifecycle costs (LCC) after intervention.

Moreover, the multi-criteria analysis offers a range of solutions grouped into two main strategies. Strategy A consists of interventions with excellent energy performance, medium LCC costs, and high investment costs. Strategy B consists of medium energy performance, low LCC costs, and medium investment costs. From the financial point of view, the different intervention strategies correspond to two management alternatives related to the owners' faculty for investing funds in the initial period.
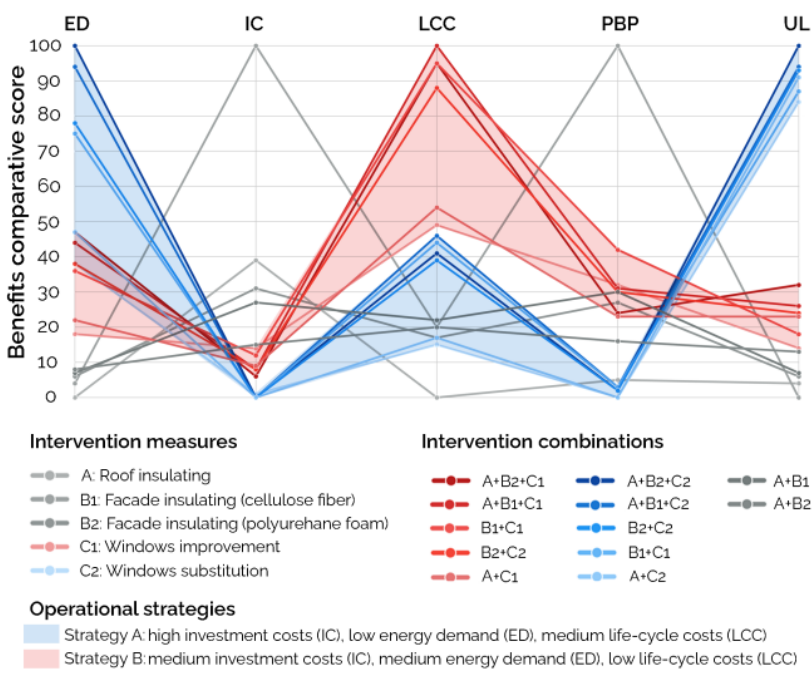

Figure 4. Multi-criteria analysis comparing different energy improvement solutions. Scores are expressed in terms of benefits from 0 to 100 with respect to five parameters.

Two significant interventions combinations could be selected respectively for the two intervention strategies and compared with the current condition of the building. Combination $\mathrm{A}+\mathrm{B} 2+\mathrm{C} 1$ (Strategy A) would result in a $73 \%$ ED decrease and a $24 \%$ LCC decrease. Combination A+B2+C2 (Strategy B) would result in 55\% energy demand decrease and $43 \%$ LCC decrease with respect to the current status of the building. Moreover, combination $\mathrm{A}+\mathrm{B} 2+\mathrm{C} 2$ requires $55 \%$ less IC than combination $\mathrm{A}+\mathrm{B} 2+\mathrm{C} 1$. In this research phase, the authors suggest interpreting these numbers as qualitative scores to compare different intervention solutions due to the so-called building energy performance gap (de Wilde, 2014), which means that the actual building energy performance does not often match predictions.

\section{FUTURE DEVELOPMENTS}

The realized models and analyses represent the first steps for creating a dynamic, accessible, and sharable Digital Twin. The installation of an IoT network inside the building and its integration with BIM and BEM data would perform the dynamic monitoring of the building's conditions in a common data environment. Such DT could be used for three primary purposes during the operational phase: BEM calibration, energy consumption reduction, and real-time performance evaluation.

\subsection{DT for energy model calibration}

The deployment of smart energy meters and IoT sensors has highlighted significant discrepancies between the simulated and the measured energy use. This is because buildings suffer from the so-called building-energy performance gap. This gap can occur at the design, construction and operation stages. For instance, the realized construction solution can deviate from those specified in the design and result in lower performance, or occupant behavior and thermal comfort levels can deviate from assumptions during the building lifecycle.

Therefore, it is fundamental to calibrate the ante-operam BEM model before performing design solutions simulations. This operation can be very challenging, depending on the complexity of the building (Chong et al., 2021). However, it is essential since it will be paid back in the renovation design phases that will be faster, cheaper and will provide better results with fewer 
uncertainties. In order to make more reliable simulations, to monitor building energy parameters through real-time data directly coming from the building and not just through deterministic simulation models could be appropriate.

\subsection{DT as a category of intervention on existing buildings}

DT could be considered an efficient intervention category for improving the energy behavior of existing buildings. This DT use would require the configuration of an intelligent system where ICT, IoT, big data, and AI are combined interacting with HBIM models to set up predictive tools for energy management and save energy consumption while satisfying the comfortable use of the building. BIM could be coupled with supervised learning techniques to investigate and produce an efficient and intelligent storage system management. Integrating AI and IoT could help asset managers predicting conditions even in real-time, automate repetitive processes, and develop innovative and efficient decision-making practices.

This approach could result in lower consumer bills, reduced impact on the natural environment, and well-performed smart asset management (Lu et al., 2020).

\subsection{DT for performance-based heritage management}

In order to pursue sustainability objectives, refurbishment interventions effectively must produce the results expected in the design phase. Therefore, results must be quantifiable in terms of performance. The ongoing digitalization of the construction industry offers a valuable chance to achieve the performancebased management of built assets. This means setting up intelligent, shared, transparent, and traceable common data environment that includes smart contracts and blockchain.

On the one hand, DTs could be used for monitoring the postintervention situation by verifying if the trend of the predicted energy parameters corresponds to the actual one detected by IoT sensors. On the other hand, DTs could allow performance-based contracts by setting performance expectations through simulation, measuring, and updating the actual state of performance, and providing recommendations for operations and maintenance through analytics. Performance-based building contracts could be legal instruments helpful to incentivize parties to deliver a building that meets targeted performance levels. Furthermore, blockchain can ensure an unchangeable and transparent digital record of transactions (Di Giuda and Pattini, 2021).

This approach could perform the evaluation of the actual performance of energy-improved heritage and activate a consistent innovation in the construction sector. It can keep the performance of the building under control and introduce the gig economy revolution (Abraham et al., 2019) in the construction sector to set up more lean and transparent practices.

\section{CONCLUSIONS}

The presented research sets up a four-step method based on the integration of HBIM and BEM models to improve the energy behavior of the Institute of Mathematics of Bologna, a valuable example of modern architecture built between 1955 and 1964.

In this paper, the first three steps of the HBIM-based workflow are illustrated, including the documental phase, the computational phase, and the predictive phase. In the first, information about the current status of the building is acquired through archival research, in-situ investigations, digital surveys, and sharing with administrators. In the second, the HBIM and BEM models are realized and integrated through open formats in the perspective of the specific information uses of energy modeling and costs scheduling and a LOK300 level of knowledge. In the third, intervention measures for the energy retrofitting of the building are investigated, and a multicriteria analysis is performed to define the optimal solutions in terms of energy, economic and financial issues. Two different financial strategies are also defined concerning the faculty of owners to invest in the initial period.

The models, procedures, and activities made in these three phases constitute the basis for realizing a Digital Twin of the building and supporting the operational phase, where DT could be used for energy model calibration, energy consumption reduction, and real-time performance evaluation.

The application of the method on a significant case study allows for systematizing a digital workflow that could be used for the information modeling of case studies with similar typological and functional characteristics. In this way, it could be possible to digitize other modern buildings belonging to the whole building stock of the University of Bologna in order to activate its performance-based management. This theme has a crucial importance in order to achieve the sustainable development of built assets from the economic, environmental and technical point of views. In addition, future repetitions of the workflow would allow for implementing an HBIM-repository (HBIR) of the recurrent technical solutions within the Italian Modern Construction. It would be an interesting tool to share knowledge about the category of listed modern buildings.

\section{REFERENCES}

Abraham, K.G., Haltiwanger, J., Sandusky, K., Spletzer, J., 2019. The Rise of the Gig Economy: Fact or Fiction? AEA Pap. Proc., 109, 357-361. doi.org/10.1257/pandp.20191039.

Bonomolo, M., Di Lisi, S., Leone, G., 2021. Building Information Modelling and Energy Simulation for Architecture Design. Appl. Sci. 11, 2252. doi.org/10.3390/app11052252

buildingSMART Italy, 2021. Guideline on the use of BIM for energy performance. Which information is exchanged, when, why, and among who?

https://www.buildingsmartitalia.org/utenti/pubblicazioni/energy -performance/ (accessed on 9 December 2021)

Castellano-Román, M., Pinto-Puerto, F., 2019. Dimensions and Levels of Knowledge in Heritage Building Information Modelling, HBIM: The model of the Charterhouse of Jerez (Cádiz, Spain). Digit. Appl. Archaeol. Cult. Herit. 14. doi.org/10.1016/j.daach.2019.e00110

Chong, A., Gu, Y., Jia, H., 2021. Calibrating building energy simulation models: A review of the basics to guide future work. Energy Build. 253, 111533.

doi.org/10.1016/j.enbuild.2021.111533

de Wilde, P., 2014. The gap between predicted and measured energy performance of buildings: A framework for investigation. Autom. Constr. 41, 40-49.

doi.org/10.1016/j.autcon.2014.02.009 
Di Giuda, G.M., Pattini, G., 2021. Transizione digitale per l'ambiente costruito. Edizioni scientifiche italiane, Napoli, Italy.

Gerber, D., Nguyen, B., Gaetani, I., 2019. Digital Twin. Towards a Meaningful Framework.

https://www.arup.com/perspectives/publications/research/sectio n/digital-twin-towards-a-meaningful-framework (accessed on 9 December 2021)

Grieves, M.W., 2019. Virtually Intelligent Product Systems: Digital and Physical Twins, in Flumerfelt, S., Schwartz, K.G., Mavris, D., Briceno, S., Complex Systems Engineering: Theory and Practice. AIAA, Reston, USA, pp. 175-200. doi.org/10.2514/5.9781624105654.0175.0200

Hong, Y., Ezeh, C.I., Deng, W., Hong, S.-H., Ma, Y., Tang, Y., Jin, Y., 2021. Coordinated energy-environmental-economic optimisation of building retrofits for optimal energy performance on a macro-scale: A life-cycle cost-based evaluation. Energy Convers. Manag. 243, 114327.

doi.org/10.1016/j.enconman.2021.114327

ENEA Agenzia nazionale per le nuove tecnologie, l'energia e lo sviluppo economico sostenibile, 2017. Linee guida per Progetti di Riqualificazione Energetica della PA Centrale (PREPAC).

Inglese, R., Ferrari, L., 2010. Giovanni Michelucci: i nuovi Istituti di Matematica e Geometria. Aterisco, Bologna, Italy.

Intelligent Energy Europe (IEE), 2012. Typology Approach for Building Stock Energy Assessment. Main Results of the TABULA project.

https://episcope.eu/fileadmin/tabula/public/docs/report/TABUL A_FinalReport_AppendixVolume.pdf (accessed on 9 December 2021)

Iori, T., 2014. SIXXI 1: storia dell'ingegneria strutturale in Italia. Gangemi, Roma.

Jouan, P., Hallot, P., 2019. Digital Twin: a HBIM-based methodology to support preventive conservation of historic assets through heritage significance awareness. Int. Arch. Photogramm. Remote Sens. Spat. Inf. Sci. XLII-2/W15, 609-615. doi.org/10.5194/isprs-archives-XLII-2-W15-609-2019

Korro Bañuelos, J., Rodríguez Miranda, Á., Valle-Melón, J.M., Zornoza-Indart, A., Castellano-Román, M., Angulo-Fornos, R., Pinto-Puerto, F., Acosta Ibáñez, P., Ferreira-Lopes, P., 2021. The Role of Information Management for the Sustainable Conservation of Cultural Heritage. Sustainability 13, 4325. doi.org/10.3390/su13084325

Kreider, R., Messner, J.I., 2013. The Uses of BIM. Classifying and Selecting BIM Uses.

http://home.fa.utl.pt/ franc/2020/the_uses_of_bim.pdf

(accessed on 9 December 2021)

Lee, D., Lee, S.H., Masoud, N., Krishnan, M.S., Li, V.C., 2021. Integrated digital twin and blockchain framework to support accountable information sharing in construction projects. Autom. Constr. 127, 103688.

doi.org/10.1016/j.autcon.2021.103688

Lu, Q., Parlikad, A.K., Woodall, P., Don Ranasinghe, G., Xie, X., Liang, Z., Konstantinou, E., Heaton, J., Schooling, J., 2020. Developing a Digital Twin at Building and City Levels: Case
Study of West Cambridge Campus. J. Manag. Eng. 36, 05020004. doi.org/10.1061/(ASCE)ME.1943-5479.0000763

Mochi, G., Predari, G., 2012. La costruzione moderna a Bologna: 1875-1915 : ragione scientifica e sapere tecnico nella pratica del costruire in cemento armato. Bruno Mondadori, Milano, Italy.

Murphy, M., McGovern, E., Pavia, S., 2009. Historic building information modelling (HBIM). Struct. Surv. 27, 311-327. doi.org/10.1108/02630800910985108

Osello, A., Lucibello, G., Morgagni, F., 2018. HBIM and virtual tools: a new chance to preserve architectural heritage. Build. Open Access J. Built Environ. 8, 1-12.

Poretti, S., 2008. Modernismi italiani. Architettura e Costruzione del Novecento. Gangemi Editore, Roma, Italy.

Porsani, G.B., Del Valle, K., Sanchez-Ostiz Gutièrrez, A., Bandera, C.F., 2021. Interoperability between Building Information Modelling (BIM) and Building Energy Model (BEM). Appl. Sci. 11(5), 2167. doi.org/10.3390/app11052167

Succar, B., Poirier, E., 2020. Lifecycle information transformation and exchange for delivering and managing digital and physical assets. Autom. Constr. 112, 103090. doi.org/10.1016/j.autcon.2020.103090

Tao, F., Zhang, M., Nee, A.Y.C., 2019. Digital twin driven smart manufacturing. Academic Press, an imprint of Elsevier, London, United Kingdom.

UN General Assembly, 2015. Transforming our world: the 2030 Agenda for Sustainable Development. https://sdgs.un.org/2030agenda (accessed on 9 December 2021)

Zevi, B., 2004. Storia dell'architettura moderna. Einaudi, Torino, Italy. 